\title{
Optimal configuration of distributed power flow controller to enhance system loadability via mixed integer linear programming
}

\author{
Jianfeng DAI ${ }^{1}$, Yi TANG ${ }^{1} \mathbb{D}$, Yuqian LIU $^{1}$, Jia NING ${ }^{1}$, \\ Qi WANG ${ }^{1}$, Ninghui $\mathrm{ZHU}^{2}$, Jingbo $\mathrm{ZHAO}^{3}$
}

\begin{abstract}
Increasing energy consumption has caused power systems to operate close to the limit of their capacity. The distributed power flow controller (DPFC), as a new member of distributed flexible AC transmission systems, is introduced to remove this barrier. This paper proposes an optimal DPFC configuration method to enhance system loadability considering economic performance based on mixed integer linear programming. The conflicting behavior of system loadability and DPFC investment is analyzed and optimal solutions are
\end{abstract}

CrossCheck date: 28 April 2019

Received: 22 December $2017 /$ Accepted: 28 April 2019/Published online: 29 August 2019

(c) The Author(s) 2019

$\triangle$ Yi TANG

tangyi@ seu.edu.cn

Jianfeng DAI

daijianfeng2012@126.com

Yuqian LIU

liuyuqian001@126.com

Jia NING

ningjia@seu.edu.cn

Qi WANG

wangqi@seu.edu.cn

Ninghui ZHU

zhuninghui@163.com

Jingbo ZHAO

L20050412@163.com

1 College of Electrical Engineering, Southeast University, Nanjing 210096, China

2 China EPSI Science \& Technology Company, Beijing 102200, China

3 State Grid Jiangsu Electric Power Company Research Institute, Nanjing 211100, China calculated. Thereafter, the fuzzy decision-making method is implemented for determining the most preferred solution. In the most preferred solution obtained, the investment of DPFCs is minimized to find the optimal number, locations and set points. Simulation results on the IEEERTS79 system demonstrate that the proposed method is effective and reasonable.

Keywords Distributed power flow controller (DPFC), Optimal configuration, System loadability, Mixed integer linear programming

\section{Introduction}

With the rapid increase in load demand and renewable energy generation, power systems urgently need to regulate power flow accurately. Due to the lack of capital resources in transmission facilities, several transmission lines are operating close to their thermal limits. The congestion will lead to a deterioration of loadability, which affects the safe and stable operation of the power grid. Thus, the maintenance of line power flow within predefined limits and the improvement of loadability are major tasks for power system planners. One option to enhance loadability is to construct new transmission lines. An alternative is to fully utilize the capacity of the existing transmission infrastructure [1]. Environmental and economic constraints make the construction of new lines arduous in urban areas. With the advent of power electronics technology, utilization of flexible AC transmission system (FACTS) devices has become one of the important options to improve power grid stability and loadability [2-4] by adjusting some system parameters such as line impedance, bus voltage and angle. Although various types of FACTS devices have been 
investigated theoretically for decades, high initial investment cost and reliability concerns are the major factors that limit their widespread adoption.

To alleviate this barrier, the concept of distributed FACTS (D-FACTS) is proposed in [5]. It can be attached directly to lines by utilizing cheap low-rated power components, which reduces the manufacturing cost and improves the convenience of the installation. As a result, power flow control or voltage regulation requirements can be satisfied in different operation modes by adjusting the number of D-FACTS devices installed on the relevant lines. It is expected that D-FACTS devices will play a good role in the transmission network after passing the required research stage. In [6], a distributed power flow control scheme for a power grid is designed by taking a series reactive power compensation device as an example, and its significant impact on power grid utilization and system reliability is analyzed. Reference [7] gives a distributed series impedance (DSI) and a distributed static series compensator (DSSC). These can be clipped on to an existing power line and change the impedance of the lines dynamically and statically to control the power flow. Further, the impact of installing D-FACTS devices on power system are studied in [8] by studying the linear sensitivities of power system quantities such as voltage magnitude, voltage angle, bus power injections, line power flow, and real power losses with respect to line impedance.

Reference [9] presents an overview on the D-FACTS controller and demonstrates the feasibility of enhancing power flow controllability. It categorizes the D-FACTS operation mode into two main classes: by-pass mode and compensation mode. In the by-pass mode, the D-FACTS controller bypasses the power under the fault conditions by using the parallel connected back-to-back thyristor switches. In the compensation mode, the D-FACTS controller achieves power flow control by compensating transmission line impedance. A new type of D-FACTS called distributed power flow controller (DPFC) is proposed in [10], and its compensation mode can be divided into series reactor mode and reactive voltage injection mode according to the selection of the module switch and the state change of the composite switch. A multi-timescale coordinated scheduling model with DPFC to minimize wind power spillage is investigated in [10].

However, these benefits of FACTS or D-FACTS devices are dependent on their number, location and set points. Recently, research has concentrated on the optimal configuration of lumped FACTS devices. From the literature review, existing studies in this area can be divided into two categories as follows: (1) Various optimization objectives are utilized to define the optimal configuration of FACTS devices. Reference [11] presents the optimal number and location of thyristor-controlled phase shifter transformers
(TCPST) to enhance loadability via mixed integer linear programming (MILP). The optimal placement of static var compensator (SVC) and thyristor-controlled series compensators (TCSC) is investigated with the objectives of loadability maximization and installation cost minimization using multi-objective particle swarm optimization method in $[12,13]$; (2) Several FACTS devices are optimally implemented to improve system performance. References [14-17] present the effects of different types of FACTS devices on system voltage stability, power flow entropy or loadability. In addition, multi-objective optimization placement of multi-type FACTS devices is investigated in [18-20] based on intelligent optimization algorithms.

Compared with the lumped FACTS devices, little effort has been devoted to the configuration of D-FACTS devices. In [21], the optimal placement of DSSCs is presented by investigating the sensitivity of real power loss with respect to line impedance. However, this method, which decouples the location and capacity of devices, cannot always achieve the optimal objective. Reference [22] presents the optimal locations of DSSCs for enhancing the loadability and system reliability for a specific number of devices, but the optimal installation number of DSSCs is not considered. In addition, performance enhancement of a hybrid AC/DC microgrid based on optimal configuration of D-FACTS is introduced in $[23,24]$. The microgrid operation performance mentioned includes stabilizing the bus voltage, reducing the feeder losses, improving the power factor and mitigating the harmonic distortion using the DFACTS devices. These are studied in [23], and D-FACTS cooperation in renewable integrated microgrids based on a linear multi-objective approach to contribute to a higher penetration of photovoltaics is proposed in [24].

However, none of the aforementioned studies take into account the conflict between investment and loadability enhancement when determining the optimal D-FACTS device configuration. Actually, the installation of D-FACTS devices in power systems is an investment issue, and it is necessary for any new installation of D-FACTS devices to be very well planned.

This paper develops an optimization model of the DPFC and presents its optimal configuration method based on mixed integer linear programming. The optimization problem to maximize the system loadability for a specific number of DPFC is formulated and a number of optimal solutions are computed. The fuzzy decision-making method is used to select the most preferred solution by power planners considering their preference. Thereafter, for the most preferred solution obtained, a minimal number of DPFC is optimally selected to meet this requirement.

The rest of this paper is organized as follows. The introduction and mathematical model of DPFC are 
presented in Section 2. In Section 3, the optimization problem is formulated. In Section 4, numerical results are used to demonstrate the effectiveness of the proposed method. Conclusions are presented in Section 5.

\section{Structure and mathematical model of DPFC}

\subsection{Structure of DPFC}

The DPFC can be directly installed on the conductor. This makes it overcome many drawbacks of existing lumped FACTS technologies. The installation and electromechanical concept diagram are shown in [10]. The remarkable benefits are as follows: (1) ability to match the investment with the actual increase of load demand; (2) ability to plan to adjust the locations according to the change of system operation modes; (3) flexible installation does not require an additional footprint; (4) avoidance of high voltage insulation, thus reducing manufacturing costs.

Figure 1 shows the schematic representation of the DPFC that consists of a single turn transformer (STT), a low-power single-phase inverter, a communication module and a composite switch. The DPFC installed on lines is controlled by the control center deployed in the substation. The main task of the control center is to obtain DPFC operation status and then pass system commands to the control module in the DPFC. System commands for gradual changes are received from the control center using a wireless technique. The inverter and control module are self-powered by induction from the conductor. The composite switch contains a normally closed electromechanical switch and a thyristor switch. The electromechanical switch is used to bypass the DPFC if the transmission line current is relatively small. The thyristor switch is used to ensure that the DPFC is bypassed quickly if a fault is detected by the current feedback signal.

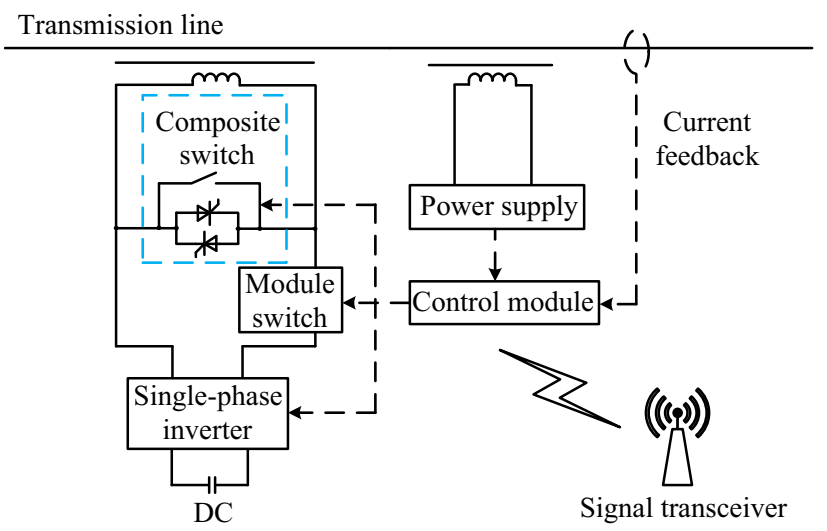

Fig. 1 Schematic representation of a DPFC

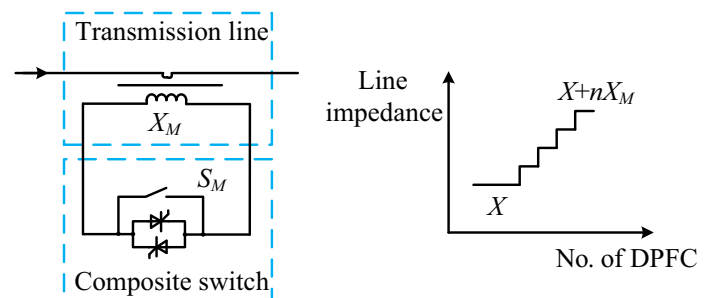

(a)

(b)

Fig. 2 Circuit schematic and corresponding operation feature of DPFC cooperating in SRM

\subsection{Control modes of DPFC}

Overall operation and control modes of the DPFCs are coordinated from the control center to realize a variety of system operation targets. A DPFC can be controlled to operate in series reactor mode (SRM) or voltage injection mode (VIM). Control modes of the DPFC and the corresponding operation features are as follows.

\subsubsection{SRM}

In SRM, a constant reactance is inserted into the transmission line.

Figure 2 clearly shows the circuit schematic of the DPFC when it operates in SRM. The single-phase inverter is not activated. With the normally closed electromechanical switch $S_{M}$ closed, the STT is bypassed and only a minimal level of reactance, corresponding to the STT leakage reactance, is inserted into the line. Switch $S_{M}$ can be opened to inject an impedance corresponding to the STT magnetizing inductance $X_{M}$. If there are $n$ DPFCs installed on transmission lines, the line impedance will increase to $n X_{M}$ as shown in Fig. 2. $X$ is the reactance of the transmission line. However, the STT magnetizing inductance is relatively small (about $50 \mu \mathrm{H}$ ) considering its weight or capacity, and the influence of the DPFC operating in SRM on power flow is limited. Thus, it is usually considered as the backup mode.

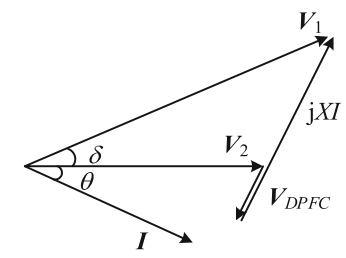

(a) Capacitive compensation

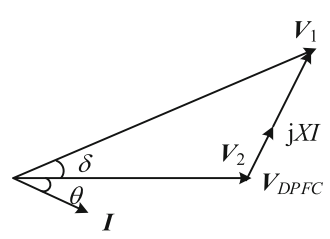

(b) Inductive compensation
Fig. 3 Phasor diagram of injecting voltage of DPFC 


\subsubsection{VIM}

In VIM, a desired quadrature voltage is injected to the transmission line to simulate positive or negative reactance.

Figure 3 shows the DPFC series injection voltage phasor diagram when the inverter is activated. $\boldsymbol{V}_{1}$ and $\boldsymbol{V}_{2}$ are the voltage vector of the sending and receiving end buses; $\boldsymbol{V}_{D P F C}$ is the voltage injected by a DPFC; $\boldsymbol{I}$ is the line current vector; $I$ is the line current amplitude; $X$ is the line reactance; $\delta$ is the phase angle difference between the two ends of the line; $\theta$ is the phase angle difference between the voltage $\boldsymbol{V}_{2}$ and current $\boldsymbol{I}$.

The operation feature of the DPFC is similar to the static synchronous series compensator (SSSC). The inverter can operate in the following compensation modes:

1) Capacitive compensation mode: the phase of injected voltage lags the line current by $90^{\circ}$, which increases the amplitude of the line current and transmitted power.

2) Inductive compensation mode: provides a desired leading quadrature voltage, thereby the transmitted power can be reduced.

It can be seen that a higher level of flexibility for improving system loadability can be obtained if the DPFC operates in VIM because the single-phase inverter can emulate any voltage or impedance within its range. This provides higher granularity of power flow control. Thus, this paper develops the mathematical model of DPFC operating in VIM. In addition, the application of DPFC is more suitable for a relatively low voltage grid than an extra high voltage (EHV) power grid. This is mainly because the low-voltage power grid is denser, the transmission length between nodes is shorter, and the electrical connection is closer, and thus the unit capacity DPFC has a better system loadability.

\subsection{Linear mathematical model of DPFC}

Figure 4 presents the circuit model of a DPFC deployed on a transmission line. As mentioned in Section 2.2, a DPFC can inject a desired quadrature voltage into the transmission line. Therefore, the relationship of line current and the voltage injected by a DPFC can be described as:

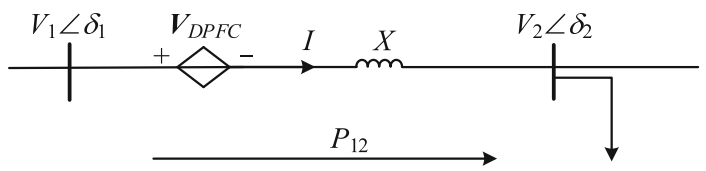

Fig. 4 Circuit model of a transmission line with DPFC
$\boldsymbol{V}_{D P F C}= \pm \mathrm{j}\left|V_{D P F C}\right| \frac{\boldsymbol{I}}{|\boldsymbol{I}|}$

where $\left|V_{D P F C}\right|$ is the voltage amplitude injected by a DPFC; and $\boldsymbol{I} /|\boldsymbol{I}|$ is the unit vector in the direction of the line current vector.

The series injected voltage clearly has an impact on the control of line power flow. The real power flow $P_{12}$ along a transmission line deployed with a DPFC can be described as:

$P_{12}=\frac{V^{2}}{X} \sin (\delta) \pm \frac{V\left|V_{D P F C}\right|}{X} \cos \left(\frac{\delta}{2}\right)$

where $V$ is the voltage amplitudes of buses 1 and 2 .

Since a DPFC is used to control real power flow and is installed in the high voltage level transmission network, the DC power flow model is used for the mathematical formulation of the DPFC. In the DC model, the voltage amplitudes of all buses are assumed equal to 1 p.u., and voltage phase angle differences are assumed to be extremely small. Therefore, the active power flow given in (2) can be simplified to:

$P_{12}=\frac{\delta}{X}+\frac{V_{D P F C}}{X}$

Figure 5 shows the variation of power flow along a transmission line that can be achieved by injecting a positive or negative voltage.

It can be seen from Fig. 5 that the injected voltage of DPFC is a continuous value and could change within its bounds. Therefore, a DPFC can either operate in inductive compensation mode (for $V_{D P F C}<0$ ) or capacitive compensation mode (for $V_{D P F C}>0$ ) which is more conductive to accurate control of power flow.

Using the linearization model of a DPFC in (3), the injected voltage of the DPFC can be embedded in the

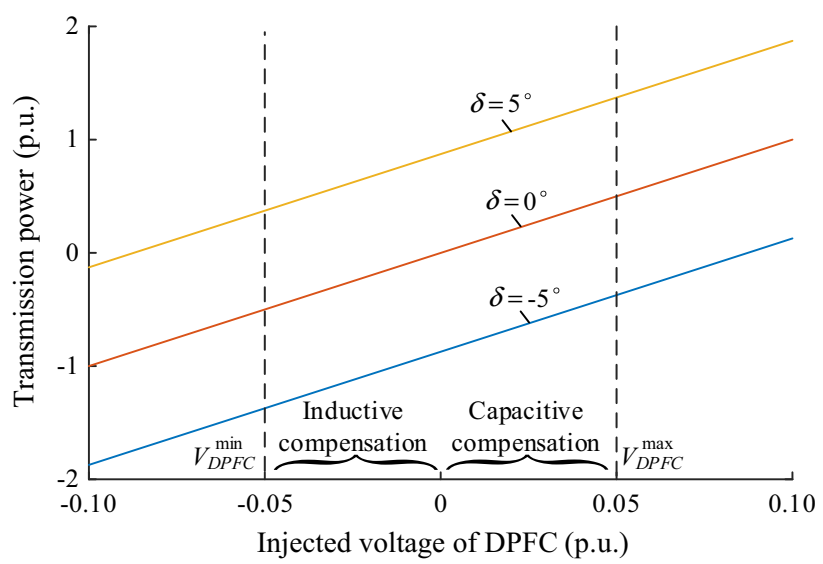

Fig. 5 Variation of transmission line power flow by quadrature voltage injection 
optimization model as a continuous control variable and solved by a linear programming solver.

\section{Problem formulation}

As an index to indicate the level of steady-state transfer capacity of a transmission system, the system loadability, representing the maximum power that can be transferred between generators and loads before security constraints, is violated in system planning. The optimization problem of obtaining maximum loadability is formulated in terms of a single unknown scalar parameter $\alpha$, whose value is expected to be maximized. In general, $\alpha$ is the load factor and represents the uniform growth of all loads in the system, and can be defined by the following equations:

$\left\{\begin{array}{l}P_{d i}=\alpha P_{d i}^{0} \\ Q_{d i}=\alpha Q_{d i}^{0}\end{array}\right.$

where $P_{d i}^{0}$ and $Q_{d i}^{0}$ are the initial active and reactive loads at bus $i$, respectively; and $P_{d i}$ and $Q_{d i}$ are the modified values, respectively. Thus, the active and reactive power balance equations on bus $i$ are expressed as:

$$
\left\{\begin{array}{l}
P_{g i}-\sum_{j=1}^{n} P_{i j}=\alpha P_{d i}^{0} \\
Q_{g i}-\sum_{j=1}^{n} Q_{i j}=\alpha Q_{d i}^{0}
\end{array}\right.
$$

where $P_{g i}$ and $Q_{g i}$ are the active and reactive power outputs of generating unit $i$, respectively; and $P_{i j}$ and $Q_{i j}$ are the transmitted powers from bus $i$ to bus $j$, respectively.

System loadability is affected by some system constraints such as distribution of its generation resources and thermal limits of the transmission network. Reference [25] has observed that loadability cannot be improved when the installation number of FACTS devices comes to a specific value. That is, the marginal loadability improvement is affected by the number of devices. The deployment of more devices can increase the flexibility in improving loadability; however, it may reduce the economy of FACTS devices. Thus, studies that investigate the configuration of DPFCs should address the problem of how to compromise between loadability and investment.

In order to solve the above problem, the optimization problem that achieving the "most effective" solution with minimal number of DPFCs is formulated. The efficiency on the loadability for different numbers of DPFCs has been quantified in Section 3.1. A fuzzy decision-making tool is used to identify the most preferred solution in Section 3.2. In the last section, optimal DPFC configuration is determined to achieve this predefined solution.

\subsection{Maximizing system loadability}

A standard optimization problem of maximizing the loadability is presented as follows:

$\max \alpha$

s.t.

$F_{k}-B_{k}\left(\theta_{n}-\theta_{m}\right)=0$

$\sum_{k \in \chi^{+}(n)} F_{k}-\sum_{k \in \chi^{-}(n)} F_{k}+\sum_{g \in g(n)} P_{g}=\alpha P_{d n}$

$P_{g}^{\min } \leq P_{g} \leq P_{g}^{\max }$

$-F_{k}^{\max } \leq F_{k} \leq F_{k}^{\max }$

$-\pi \leq \theta_{n} \leq \pi$

where $F_{k}$ is the power flow of transmission line $k ; B_{k}$ is the susceptance of line $k ; \theta_{n}$ and $\theta_{m}$ are the voltage angles at buses $n$ and $m$, respectively; $P_{g}$ is the active power output of unit $g ; P_{d n}$ is the load at bus $n ; k$ is the index of transmission lines; $g$ is the index of generators; $\chi^{+}(n)$ is the set of lines specified as to node $n ; \chi^{-}(n)$ is the set of lines specified as from node $n ; g(n)$ is the set of generators connected to node $n ; P_{g}^{\max }$ and $P_{g}^{\min }$ are the upper and lower active power limits on the generating unit $g$, respectively; and $F_{k}^{\max }$ is the thermal limit of transmission line $k$.

Equation (6) represents that the corresponding objective function is to maximize the system loadability. Equations (7)-(8) represent the active power flows on transmission lines and active power balance equations. Equations (9)-(11) represent the lower and upper limits on generator real power outputs, line power flows and bus voltage angles.

The introduction of a DPFC adds new control variables to the system. As mentioned above, DPFCs have significant impact on power flows by injecting a quadrature voltage. Using the proposed model for the DPFCs in Section 2.3, the power flow on transmission lines implemented with DPFCs can be described as:

$F_{k}-B_{k}\left(\theta_{n}-\theta_{m}\right)-B_{k} V_{q k}=0$

where $V_{q k}$ is the voltage injected by DPFC installed on the line $k$.

Since DPFCs can be attached directly to the transmission lines, the capability of transmission lines to withstand DPFC weight should be considered. Therefore, the number of DPFCs installed on each line should be restricted. Thus, DPFC physical and operation constraints are presented as:

$N_{k, \min } u_{k} \leq N_{k} \leq N_{k, \max } u_{k}$ 
$3 \sum_{k} N_{k} \leq N_{T}$

$N_{k} V_{q k, \text { min }} \leq V_{q k} \leq N_{k} V_{q k, \text { max }}$

where $N_{k}$ is the number of DPFC installed on line $k ; N_{k, \min }$ and $N_{k \text {,max }}$ are the minimum and maximum number of DPFCs installed on the line $k$, respectively; $u_{k}$ is the binary parameter indicating whether a DPFC can be installed on line $k ; N_{T}$ is the maximum total number of DPFCs available to be installed in the system; and $V_{q k, \text { min }}$ and $V_{q k \text {,max }}$ are the lower and upper limits on voltage injected by a DPFC installed on the line $k$, respectively.

Equation (13) expresses the lower and upper bound on the number of DPFCs attached to each transmission line. In (13), $u_{k}$ is a binary parameter. In this way, setting $u_{k}$ to one indicates that a DPFC is allowed to be installed on line $k$. Its value will be determined by the planners according to the actual operation conditions of each line. As a DPFC can be attached directly to the transmission line, $N_{k, \max }$ is affected by the length of the line, the bearing capacity of the tower and the weight of the DPFC. The limit on the total number of DPFCs or investment resources is defined in (14). The lower and upper bounds on injected voltages of a group of DPFCs deployed on line $k$ are given by (15). These are related to the number of DPFCs installed on the line. $V_{q k, \max }$ and $V_{q k, \min }$ are determined by the capacity of the DPFC, the voltage level and the thermal limit of the line. They can be expressed as:

$V_{q k, \max }=S_{D P F C} / I_{k, \max }$

$V_{q k, \min }=-S_{D P F C} / I_{k, \max }$

where $S_{D P F C}$ is the capacity of the DPFC; and $I_{k, \max }$ is the maximum current of the transmission line $k$.

In summary, the optimization problem to enhance the loadability with DPFCs is presented in (6), (8)-(15). The optimization process implemented to evaluate the efficiency of DPFCs on the loadability can be presented as follows:

Step 1 Initialize $N_{T}=0$, obtain the initial system loadability without DPFCs.

Step 2 Increase $N_{T}=N_{T}+3$, calculate the optimal value of the system loadability for the given number of DPFCs.

Step 3 This loop continues until the maximum value of loadability does not increase even if more DPFCs are added.

\subsection{Fuzzy decision-making tool}

After increasing the total number of DPFCs on the system, the conflicting behavior between system loadability and investment of DPFCs is obtained. Thereafter, the power planners need to make a compromise between loadabilty and investment and choose the most preferred solution according to their preference. In this section, the fuzzy decision-making method as a valid approach is used, and linear membership functions are defined for evaluating the system loadability and investment of DPFCs. The system loadability and investment of DPFCs in the $i^{\text {th }}$ solution are formulated as:

$f_{1, i}=\frac{\alpha_{i}-\alpha^{\min }}{\alpha^{\max }-\alpha^{\min }}$

$f_{2, i}=\frac{C_{D P F C}^{\max }-C_{D P F C . i}}{C_{D P F C}^{\max }-C_{D P F C}^{\min }}$

where $\alpha^{\max }$ and $\alpha^{\min }$ are the maximum and minimum loadabilities; and $C_{D P F C}^{\max }$ and $C_{D P F C}^{\min }$ are the maximum and minimum investments of DPFCs in these solutions. Thus, the overall satisfaction level of $i^{\text {th }}$ solution can be described as:

$f_{i}=\left(w_{1} f_{1, i}+w_{2} f_{2, i}\right)$

where $w_{1}$ and $w_{2}$ are the weight values assigned to $f_{1}$ and $f_{2}$. These weight values can be selected by the power planners according to the importance of the economic and technical aspects of different systems. Therefore, the solution with the maximum overall satisfaction level would be the most preferred solution, and the system loadability in this solution is defined as the optimal point.

\subsection{Minimizing DPFC investment}

With the proposed approach, the most preferred loadability $\alpha_{o p t}$ will be found. To achieve this system loadability and avoid the redundant deployment of DPFCs, the total investment or the total number of DPFCs is minimized. Therefore, the problem is formulated as:

$\min \sum_{k} N_{k}$

This optimization model needs to satisfy the predefined loadability, and it is subject to (8)-(15). An additional constraint that maintains the predefined optimal loadability is described as:

$\sum_{k \in \chi^{+}(n)} F_{k}-\sum_{k \in \chi^{-}(n)} F_{k}+\sum_{g \in g(n)} P_{g}=\alpha_{o p t} P_{d n}$

The DPFC placement strategy may not be unique after being optimized in this stage. The reason is that the installation cost coefficients of each line are assumed to be equal in (21). Therefore, a unique DPFC placement can be obtained by adopting differentiated coefficients according to the actual situation. 


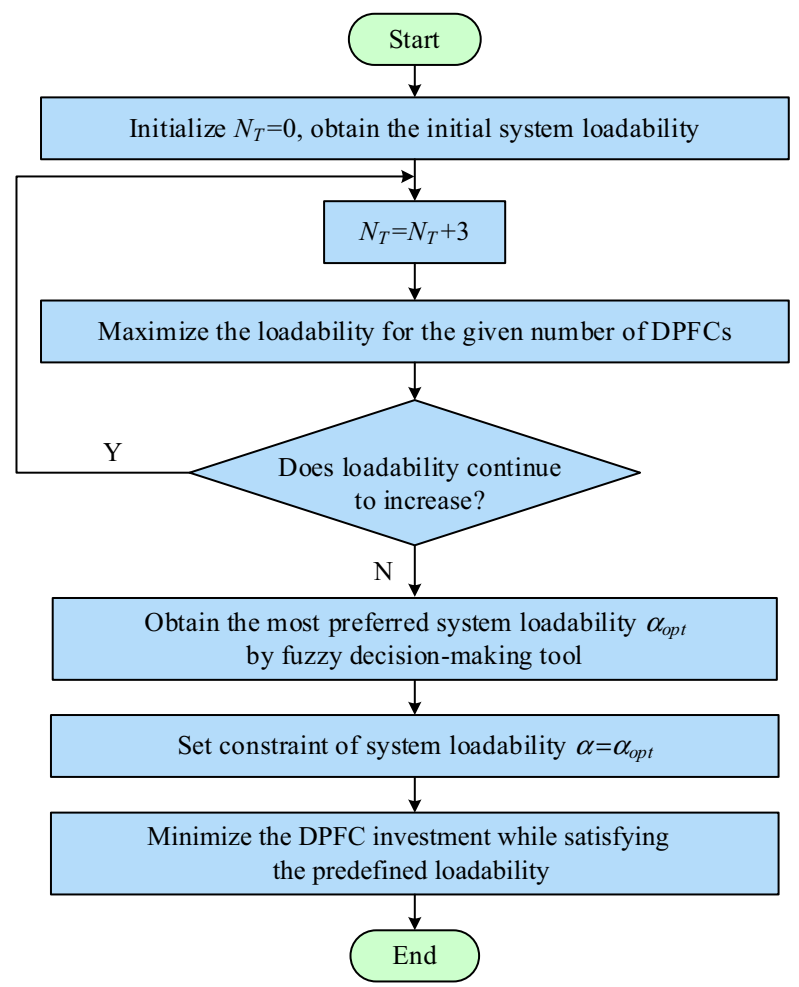

Fig. 6 Flowchart of the proposed method

It is worth mentioning that system loadability is also affected by other factors such as $S_{D P F C}$ and $N_{k, \max }$ which influence the ability to control the power flow of DPFCs installed on a specific transmission line. Thus, these detailed analyses are presented in Section 4.2.

Figure 6 shows the flowchart of the proposed method. The above MILP problem can be modeled in MATLAB and solved using optimization toolbox.

\section{Case studies}

To analyze the effect of DPFCs on system loadability and verify the correctness and validity of the proposed method, the IEEE-RTS79 system is chosen as the test system. The voltage level of transmission lines is $230 \mathrm{kV}$ and $138 \mathrm{kV}$. The total active network loads are $2850 \mathrm{MW}$, and the complete data of this test system can be obtained from [26]. To reach the network loadability limit, the transmission limits of all lines in the system are reduced by $50 \%$.

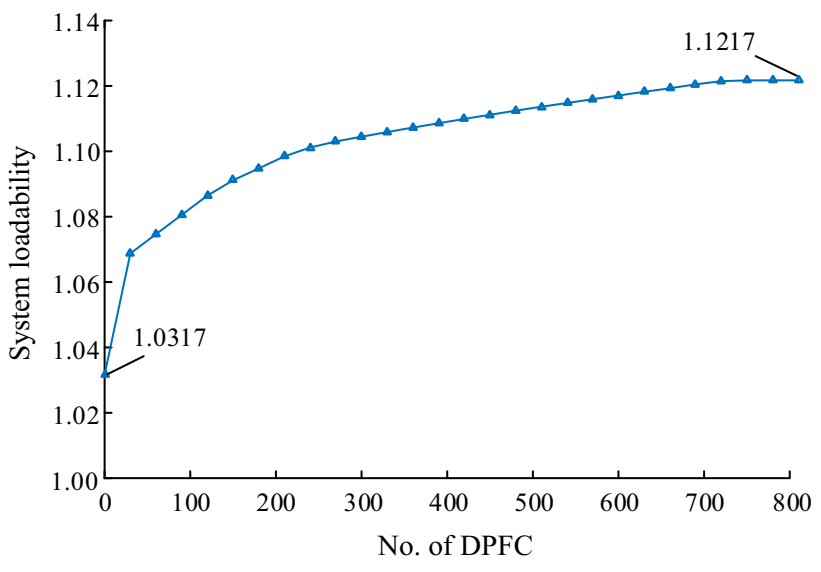

Fig. 7 Comparison of DPFC placement in two cases

Since the capacity of a DPFC is set to $70 \mathrm{kVA}$, injected voltages of DPFCs installed on different transmission lines are shown in Table 1. is the injected voltage of DPFC, $V_{\mathrm{i}}^{\prime}$ is the injected voltage of per unit.

The maximum and minimum numbers of DPFCs allowed to be installed on a conductor of a transmission line must be specified. Since DPFCs can only be attached to the lines closely connected to the strain tower and there is approximately one strain tower per mile of a transmission line, the maximum number of DPFCs installed on a transmission line is assumed to be equal to one per mile per conductor based on the weight of DPFCs and the distribution of strain towers. The minimum number of DPFCs on each line is assumed equal to zero.

\subsection{Optimal placement of DPFC}

By increasing the total number of DPFCs in the system, the conflicting behavior of loadability and investment of a DPFC is shown in Fig. 7. The maximum attainable loadability of this system is at $\alpha^{\max }=1.1217$ with approximately 810 DPFCs, which is equal to $2850 \times 1.1217=3196.84 \mathrm{MW} . \alpha^{\max }=1.0317$ has been obtained which is the maximum system loadability without DPFCs. It is noticeable that the more system loadability is achieved, the more DPFCs are applied. Thus, it is necessary to compromise between loadability and investment.

Therefore, $w_{1}$ and $w_{2}$ are both set to 0.5 to compromise between loadability and investment in a DPFC. According to the adopted weight values, the optimal operation point

Table 1 Injected voltage of DPFC on transmission lines with different voltage levels

\begin{tabular}{llllll}
\hline Maximum $V_{\mathrm{i}}(\mathrm{V})$ & Maximum $V_{\mathrm{i}}^{\prime}$ (p.u.) & Minimum $V_{\mathrm{i}}(\mathrm{V})$ & Minimum $V_{\mathrm{i}}^{\prime}$ (p.u.) & Line voltage $(\mathrm{kV})$ & Line transmission limit $(\mathrm{MW})$ \\
\hline 191.22 & $2.4 \times 10^{-3}$ & -191.22 & $-2.4 \times 10^{-3}$ & 138 & 87.5 \\
111.54 & $8.4 \times 10^{-4}$ & -111.54 & $-8.4 \times 10^{-4}$ & 230 & 250 \\
\hline
\end{tabular}


Table 2 Optimal placement of DPFCs

\begin{tabular}{|c|c|c|c|c|}
\hline \multicolumn{3}{|l|}{ Line } & \multicolumn{2}{|l|}{ DPFC } \\
\hline Sending bus & Receiving bus & Length $(\mathrm{km})$ & No. per phase (set) & Injected voltage (p.u.) \\
\hline 1 & 3 & 55 & 15 & -0.0360 \\
\hline 2 & 6 & 50 & 12 & 0.0288 \\
\hline 11 & 13 & 33 & 3 & -0.0023 \\
\hline 11 & 14 & 29 & 29 & 0.0244 \\
\hline 14 & 16 & 27 & 11 & 0.0092 \\
\hline
\end{tabular}

Table 3 Optimization results for various capacity of DPFCs

\begin{tabular}{lll}
\hline $\begin{array}{l}\text { Capacity of each } \\
\text { DPFC (kVA) }\end{array}$ & $\begin{array}{l}\text { Optimal } \\
\text { loadability }\end{array}$ & $\begin{array}{l}\text { Total No. of } \\
\text { DPFCs }\end{array}$ \\
\hline 70 & 1.0985 & 210 \\
80 & 1.0979 & 180 \\
90 & 1.1004 & 180 \\
100 & 1.0987 & 150 \\
\hline
\end{tabular}

and loadability $\alpha_{o p t}=1.0985$ are found. Table 2 presents the optimal number, locations and set points of DPFCs at the most preferred system operation point after minimizing the DPFC investment. The results given in Table 2 reveal that lines 11-14 are fully deployed with DPFCs owing to the length of the lines. This observation implies the importance of this transmission line in improving system loadability. In addition, the injected voltages of DPFCs in column 5 indicate that the compensation mode of DPFCs varies from line to line. This observation implies that the power flow of these lines can either increase or decrease by controlling the injected voltage. Thus, DPFCs can enhance system loadability by improving the distribution of power flow and making full use of the transmission limits of several transmission lines.

\subsection{Sensitivity analysis}

The development of D-FACTS technology has the main features: (1) reducing the weight of devices to make the conductor bear more equipment; (2) increasing capacity that improves the performance of devices; (3) ability to be installed on higher voltage transmission networks. Therefore, considering the improvement trend of DPFCs over time, the effects of various capacity and upper bound constraints (10) on the overall results are investigated in this paper. The capacity of each DPFC is changed from $70 \mathrm{kVA}$ to $100 \mathrm{kVA}$ in three steps, and the maximum number of DPFCs that can be installed on a transmission line is changed from 1 to 4 per mile per conductor in three steps. The optimization results are presented in Table 3 and Table 4 . The total number of DPFCs is gradually reduced when the capacity of each DPFC increases. The optimal loadability indices defined by fuzzy decision-making are slightly sensitive to the capacity of the DPFC. In addition, with the increase of the maximum number of DPFCs, though the optimal loadability and total number of DPFCs have not changed in this case, the number of lines equipped with DPFC is reduced to four. As a result, it can be deduced that if the limit is loosened by the modified technology, there is no concern on the enhancement of the efficiency.

\subsection{Comparison of DPFC with lumped FACTS device}

In addition, maximum attainable loadability is also necessary for investigating some areas where it is not convenient to construct new lines. Thus, the weight values of loadability and investment of DPFCs are set to 1 and 0 in the proposed method, respectively. This paper compares the effect of lumped FACTS and D-FACTS devices on maximizing system loadability. Simulations are performed only for a single-type device, DPFC and SSSC. Figure 8 shows the improvement of the maximum loadability for different total installed capacity of two FACTS devices. In this case, SSSC is installed on lines 1-3 after optimal placement. It can be noted that the DPFCs are superior to an SSSC in enhancing loadability at the specific capacity. The reason is that the loadability is a global problem of the power grid, and SSSC is generally installed on the same line, and this cannot effectively solve the operation limit of multiple lines, and the DPFCs with the same capacity can be distributed and flexibly installed on multiple different lines. This can solve the overload problem of multiple lines, resulting in making the loadability further enhanced.

Compared with lumped FACTS devices, one of the advantages of the DPFC is that it can flexibly adjust the allocation according to the change of system operation mode. It is to be noted that the installation and debugging of the DPFC is usually performed during the planned power outage of the grid. 
Table 4 Optimization results for various maximum numbers of DPFCs

\begin{tabular}{llll}
\hline $\begin{array}{l}\text { Maximum No. of } \\
\text { DPFCs per mile per } \\
\text { conductor }\end{array}$ & $\begin{array}{l}\text { Optimal } \\
\text { loadability }\end{array}$ & $\begin{array}{l}\text { Total } \\
\text { number of } \\
\text { DPFCs }\end{array}$ & $\begin{array}{l}\text { No. of lines } \\
\text { equipped with } \\
\text { DPFCs }\end{array}$ \\
\hline 1 & 1.0985 & 210 & 5 \\
2 & 1.0985 & 210 & 4 \\
3 & 1.0985 & 210 & 4 \\
4 & 1.0985 & 210 & 4 \\
\hline
\end{tabular}

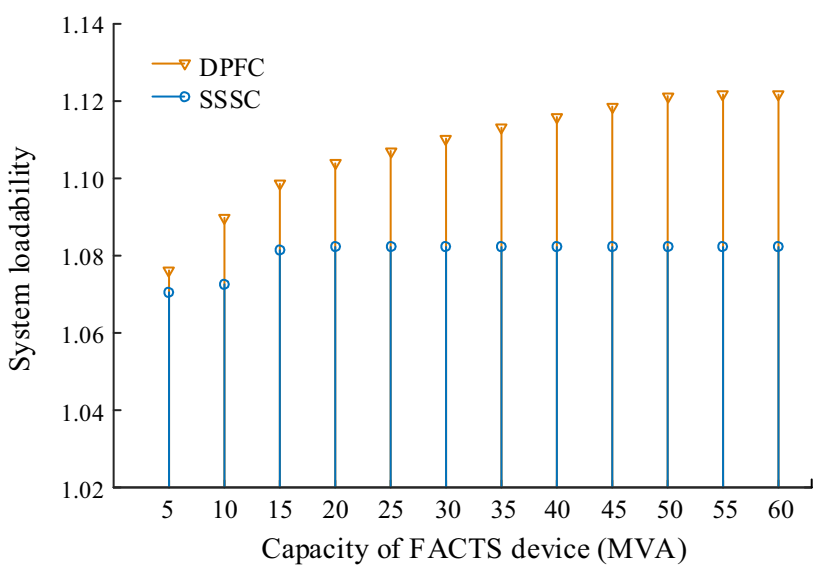

Fig. 8 System loadability for various capacity of FACTS device

Table 5 Maximum system loadability in different operation modes

\begin{tabular}{llll}
\hline $\begin{array}{l}\text { Operation } \\
\text { mode }\end{array}$ & \multicolumn{4}{l}{ Maximum attainable loadability $\alpha$} \\
\cline { 2 - 4 } & $\begin{array}{l}\text { Without FACTS } \\
\text { device }\end{array}$ & $\begin{array}{l}\text { With } \\
\text { DPFC }\end{array}$ & $\begin{array}{l}\text { With } \\
\text { SSSC }\end{array}$ \\
\hline Initial mode & 1.0317 & 1.1217 & 1.0822 \\
Mode 2 & 1.0928 & 1.1583 & 1.1085 \\
\hline
\end{tabular}

In particular, the different modes of system operation mean different load levels. To clarify the benefit of DPFCs, the following operation modes in IEEE-RTS79 system are considered for the simulation:

1) Initial mode: the initial loads data taken from [26] is chosen as the base operation mode for study.

2) Mode 2: the loads are modified to simulate the change of system operation state. Loads in $230 \mathrm{kV}$ nodes increase by $10 \%$, and other loads in $138 \mathrm{kV}$ nodes are reduced by $10 \%$. Thus, the total active network loads are 2868.6 MW. In each of these modes, the maximum attainable system loadability is calculated when integrated with different types of devices. In this paper, SSSC is chosen as the typical lumped FACTS device since the operation features of SSSC are similar to those of a DPFC.
Table 6 Configuration of DPFCs in two system operation modes

\begin{tabular}{|c|c|c|c|c|}
\hline \multicolumn{3}{|l|}{ Line } & \multicolumn{2}{|c|}{ No. of DPFC per phase } \\
\hline Sending bus & Receiving bus & Length (m) & Initial mode & Mode 2 \\
\hline 2 & 4 & 33 & 12 & \\
\hline 2 & 6 & 50 & 14 & 5 \\
\hline 3 & 9 & 31 & & 28 \\
\hline 4 & 9 & 27 & 27 & \\
\hline 8 & 9 & 43 & 42 & \\
\hline 8 & 10 & 43 & 5 & \\
\hline 11 & 13 & 33 & & 16 \\
\hline 11 & 14 & 29 & 29 & 29 \\
\hline 12 & 13 & 33 & 12 & \\
\hline 12 & 23 & 67 & 58 & \\
\hline 13 & 23 & 60 & & 22 \\
\hline 14 & 16 & 27 & 27 & 27 \\
\hline 16 & 17 & 18 & & 15 \\
\hline 16 & 19 & 16 & 16 & 16 \\
\hline 20 & 23 & 15 & & 1 \\
\hline
\end{tabular}

The optimization results on system loadability in two operation modes are presented in Table 5. It can be seen from Table 5 that with one SSSC installed on the above line, the loadability would improve by 0.0505 in the initial system operation mode, while the loadability only increases by 0.0157 in system operation mode 2 . However, system loadability increases by 0.090 and 0.0655 after optimal configuration of DPFCs.

Table 6 presents the optimal locations and numbers of DPFCs in two system operation modes. The injected voltages of DPFCs are not detailed because they are not constant values and vary from one mode to another in order to alleviate transmission congestion. The results presented in Table 6 clarify that different system operation modes cause different configurations of DPFCs among transmission lines. It can be observed that lines 2-6, 11-14, 14-16 and 16-19 are selected in two operation modes, and the last three lines are fully deployed with DPFCs owing to the length of lines. It implies that these transmission lines are important for enhancing system loadability. In addition, the results reveal that DPFCs are installed on some transmission lines only in initial mode or mode 2 . This observation shows that the lines limiting system loadability are usually different as the operation mode alters. As previously mentioned, the major advantage of DPFC is the ability to adjust its location if needed. For example, system operators can adjust their locations once or twice a year according to the change of system operation mode. Therefore, DPFC is more conducive for improving loadability. 


\section{Conclusion}

This paper presents the novel concept of a DPFC and its optimal configuration method based on mixed integer linear programming. The conflicting behavior of system loadability and investment in DPFCs is quantitatively analyzed while considering all system constraints, including physical and operation features of DPFC. The most preferred loadability is determined by a fuzzy decisionmaking method using the adopted weighting factors. At this predefined loadability, the investment of DPFCs has been minimized to determine the optimal number, locations and set points of DPFCs. The simulation results show the effectiveness of the proposed method for optimal configuration of DPFCs in a transmission system. In addition, the sensitivity analysis demonstrates that the efficiency for enhancing system loadability has been improved by the modified technology. Furthermore, the results indicate that DPFCs can distinctly enhance system loadability compared with lumped FACTS devices. The proposed method, owing to its simple computation, can determine the optimal placement of other FACTS or D-FACTS devices.

Acknowledgements This work was supported in part by the National Natural Science Foundation of China (No. 51577030) and in part by the project of State Grid Corporation of China (Research on flexible $\mathrm{AC}$ power flow control technology of transmission network based on a distributed power flow controller) (No. 8516000700).

Open Access This article is distributed under the terms of the Creative Commons Attribution 4.0 International License (http:// creativecommons.org/licenses/by/4.0/), which permits unrestricted use, distribution, and reproduction in any medium, provided you give appropriate credit to the original author(s) and the source, provide a link to the Creative Commons license, and indicate if changes were made.

\section{References}

[1] Fang RS, David AK (1999) Transmission congestion management in an electricity market. IEEE Trans Power Syst 14(3):877-883

[2] Hingorani NG (1993) Flexible AC transmission. IEEE Spectrum 30(4):40-45

[3] Maza-Ortega JM, Acha E, García S et al (2017) Overview of power electronics technology and applications in power generation transmission and distribution. J Mod Power Syst Clean Energy 5(4):499-514

[4] Ghorbani A, Ebrahimi SY, Ghorbani M (2017) Active power based distance protection scheme in the presence of series compensators. Protect Contr Modern Power Syst 2(1):7

[5] Divan DM, Brumsickle WE, Schneider RS et al (2006) A distributed static series compensator system for realizing active power flow control on existing power lines. IEEE Trans Power Del 22(1):642-649
[6] Johal H, Divan D (2007) Design considerations for series-connected distributed FACTS converters. IEEE Trans Ind Appl 43(6):1609-1618

[7] Divan D, Johal H (2007) Distributed FACTS - a new concept for realizing grid power flow control. IEEE Trans Power Electron 22(6):2253-2260

[8] Rogers KM, Overbye TJ (2008) Some applications of distributed flexible AC transmission system (D-FACTS) devices in power systems. In: Proceedings of North American power symposium, Calgary, Canada, 28-30 September 2008, 8 pp

[9] Dhaked DK, Lalwani M (2017) A review paper on a D-FACTS controller: enhanced power flow controller. Int J Adv Eng Technol 10(1):84

[10] Tang Y, Liu Y, Ning J et al (2017) Multi-time scale coordinated scheduling strategy with distributed power flow controllers for minimizing wind power spillage. Energies 10(11):1804

[11] Lima FGM, Galiana FD, Kockar I et al (2004) Phase shifter placement in large-scale systems via mixed integer linear programming. IEEE Trans Power Syst 18(3):1029-1034

[12] Chang YC (2012) Multi-objective optimal svc installation for power system loading margin improvement. IEEE Trans Power Syst 27(2):984-992

[13] Chang YC (2014) Multi-objective optimal thyristor controlled series compensator installation strategy for transmission system loadability enhancement. IET Gener Transm Distrib $8(3): 552-562$

[14] Laifa A, Medoued A (2014) Optimal FACTS location to enhance voltage stability using multi-objective harmony search. In: Proceedings of international conference on electric power and energy conversion systems, Istanbul, Turkey, 2-4 October 2013, 6pp

[15] Maza-Ortega JM, Acha E, García S et al (2017) Overview of power electronics technology and applications in power generation transmission and distribution. J Mod Power Syst Clean Energy 5(4):499-514

[16] Ghahremani E, Kamwa I (2013) Optimal placement of multipletype facts devices to maximize power system loadability using a generic graphical user interface. IEEE Trans Power Syst 28(2):764-778

[17] Ara AL, Kazemi A, Niaki SAN (2012) Multiobjective optimal location of facts shunt-series controllers for power system operation planning. IEEE Trans Power Del 27(2):481-490

[18] Ranganathan S, Kalavathi MS, Christober ARC (2016) Selfadaptive firefly algorithm based multi-objectives for multi-type facts placement. IET Gener Transm Distrib 10(11):2576-2584

[19] Basiri-Kejani M, Gholipour E (2016) Two-level procedure based on hicaga to determine optimal number, locations and operating points of svcs in isfahan-khuzestan power system to maximise loadability and minimise losses, TVD and SVC installation cost. IET Gener Transm Distrib 10(16):4158-4168

[20] Di Y, Cheng H, Ma Z et al (2018) Dynamic VAR planning methodology to enhance transient voltage stability for failure recovery. J Mod Power Syst Clean Energy 6(4):712-721

[21] Rathi A, Sadda A, Nebhnani L et al (2012) Loss minimization with D-FACTS devices using sensitivity based technique. In: Proceedings of IEEE India international conference on power electronics, Calgary, India, 6-8 December 2012, 5 pp

[22] Dorostkar-Ghamsari M, Fotuhi-Firuzabad M, Aminifar F et al (2015) Optimal distributed static series compensator placement for enhancing power system loadability and reliability. IET Gener Transm Distrib 9(11):1043-1050

[23] Abdelsalam AA, Gabbar HA, Sharaf AM (2014) Performance enhancement of hybrid AC/DC microgrid based D-FACTS. Int J Electr Power Energy Syst 63:382-393 
[24] Hamidi A, Golshannavaz S, Nazarpour D (2019) D-FACTS cooperation in renewable integrated microgrids: a linear multiobjective approach. IEEE Trans Sustain Energy 10(1):355-363

[25] Gerbex S, Cherkaoui R, Germond A (2001) Optimal location of multi-type FACTS devices in a power system by means of genetic algorithms. IEEE Trans Power Syst 16(3):537-544

[26] Subcommittee PM (1979) IEEE reliability test system. IEEE Trans Power Appl Syst PAS 98(6):2047-2054

Jianfeng DAI received his M.S. degree from Shanghai University of Electric Power, Shanghai, China, in 2015. He is currently pursuing a Ph.D. degree of Southeast University, Nanjing, China. His current research interests include operation and control of new energy integration, the application of power electronics technology in power system.

Yi TANG received his Ph.D. degree from Harbin Institute of Technology, Harbin, China, in 2006. He is currently an associate professor in Southeast University, Nanjing, China. His research interests include smart grid, power system security, power system stability analysis, renewable energy systems and cyber physical system.

Yuqian LIU received his B.S. degree in electrical engineering from Fuzhou University, Fuzhou, China, in 2015. He is currently pursuing the M.S. degree at Southeast University, Nanjing, China. His research interests include power system operation and optimization.

Jia NING received the bachelor degree in electrical engineering from Shanghai University of Electric Power, Shanghai, China, in 2012. She is currently pursuing the $\mathrm{Ph} . \mathrm{D}$. degree of Southeast University, Nanjing, China. Her research interests include demand response, renewable energy management, electric vehicle integration.

Qi WANG received his Ph.D. degree from Southeast University, Nanjing, China, in 2017. He is now a lecturer in Southeast University, Nanjing, China. His research interests include cyber physical system, power system stability analysis and power system security.

Ninghui ZHU received his Ph.D. degree from China Electric Power Research Institute, Beijing, China, in 2012. He is currently a senior engineer at China EPSI Science \& Technology Company, Beijing, China. His research interests include flexible AC transmission system.

Jingbo ZHAO received his M.S. degree from Zhejiang University, Hangzhou, China, in 2008. He is currently a senior engineer at State Grid Jiangsu Electric Power Company Research Institute, Nanjing, China. He is responsible for the analysis and control of the Jiangsu power system. 